\title{
Cutting Tool Tracking and Recognition Based on Infrared and Visual Imaging Systems using Principal Component Analysis (PCA) and Discrete Wavelet Transform (DWT) Combined with Neural Networks
}

\author{
Milad Elgargni ${ }^{1}$, Amin Al-Habaibeh ${ }^{2}$ and Ahmad Lotfi ${ }^{3}$ \\ 1,2 Advanced Design and Manufacturing Engineering Centre \\ School of Architecture, Design and the Built Environment \\ Nottingham Trent University \\ ${ }^{3}$ School of Science and Technology \\ Nottingham Trent University
}

Milad.Elgargni@ntu.ac.uk; Amin.Al-Habaibeh@ntu.ac.uk; Ahmad.Lotfi@ntu.ac.uk

\begin{abstract}
The implementation of computerised condition monitoring systems for the detection cutting tools' correct installation and fault diagnosis is of a high importance in modern manufacturing industries. The primary function of a condition monitoring system is to check the existence of the tool before starting any machining process and ensure its health during operation. The aim of this study is to assess the detection of the existence of the tool in the spindle and its health (i.e. normal or broken) using infrared and vision systems as a noncontact methodology. The application of Principal Component Analysis (PCA) and Discrete Wavelet Transform (DWT) combined with neural networks are investigated using both types of data in order to establish an effective and reliable novel software program for tool tracking and health recognition. Infrared and visual cameras are used to locate and track the cutting tool during the machining process using a suitable analysis and image processing algorithms. The capabilities of PCA and Discrete Wavelet Transform (DWT) combined with neural networks are investigated in recognising the tool's condition by comparing the characteristics of the tool to those of known conditions in the training set. The experimental results have shown high performance when using the infrared data in comparison to visual images for the selected image and signal processing algorithms.
\end{abstract}

Keywords: Image processing, PCA, Neural Network, Tool recognition, Computer software, manufacturing processes.

\section{Introduction}

Tool state recognition is important in machining processes in order to maintain the productivity of the manufacturing system and the quality of the produced products. Tool state recognition in general consists of two steps: (1) Verifying the tool's existence; and (2) detecting the tool health state (normal, severely worn or broken). In this paper tool recognition of the tool's existence and its health, normal or broken, is performed by PCA and DWT combined with neural networks using visual and infrared images. Studies found in literature on condition monitoring of tool conditions using sensor fusion of multi-sensor features reveal the general effectiveness of such approach, see for example Al-Habaibeh et al. [1-3]. They describe the implementation of sensor fusion systems for a CNC machine tool and its machining processes. The monitoring systems described have used a variety of known methods and sensors such as force, torque, vibration, acoustic emission, and temperature to monitor the machining processes and the physical condition of the machine tool. Results show that some signals are found to be useful in detecting the tool's condition while others are less sensitive. However, the work did not include the existence of the cutting tool as part of the study. And sensors that have been found useful in one occasion might not useful in 
others. Many other references found in literature have used diversity of known methods and sensors for monitoring cutting tools conditions, see for example the reviews in reference [4]. Common methods include spindle motor current signals [5], piezoelectric force sensors [6, 7], vibration [7-10], sound and acoustic emission [10-12]. The problem of the above sensors as has been argued by [4] is that the position selection of the sensor might be a problem, the sensors might be sensitive to other events and process variation, in addition to the lack of stability in during the cutting process. Therefore, researchers have attempted to use vision systems to address some of the above drawbacks if possible. In comparison, vision systems (visual and infrared) are not expected to have the same technical problems in most cases.

Many approaches and methods based on image data and image processing algorithms have been explored in this field of research for an attempt to achieving reliable tool condition monitoring. Research using visual vision systems included the use of image processing approach to detect and measure the tool flank wear area using a microscope [13], monitoring the actual tool condition based upon the edge radius of the tool [14], condition monitoring for drilling tools using high speed camera [15] and non-contact vision system for on-line monitoring of the cutting tool without removing the tool from the machine. Gradient detection algorithm with polynomial fitting has been applied in turning [16]. In most cases it has been found that the suggested techniques might be difficult to be implemented online in real industrial environments due to the required setup for the measurement processes or the systems have not been able to detect the tool conditions due to the selected image processing approach.

Condition monitoring of machining operations using image processing of infrared thermography have been limited in research, see for example [17, 18]. The use of infrared technology in other applications have been more common, see for example Al-Habaibeh et al. [19] where a mechatronic approach of using infrared thermography combined with image processing for the quality control of a laser sealing process for food containers have been presented. Recently, reference [20] has presented the sensors and signal processing technologies applied in machining processes, it is clear that insufficient research work has been covered in the area of vision systems for visual and infrared imaging to investigate tool condition monitoring (TCM).

From the above discussion it can be seen that limited research has been done in the combined area of tool recognition and identification, particularly using autonomous infrared and vision technology. There is lack in the development of a non-contact monitoring system for tool recognition and health monitoring. However, tool recognition is as important as tool wear detection. This is because the on-line and real time verification of the existence of the tool before starting machining is of high importance. However, when using two dimensional data, the complexity becomes in the selection of the most suitable image processing to enhance the classification and the decision making process. In this study, a novel implementation of DWT and PCA is considered. DWT, see for example [19-21], is normally used in signal and image processing in order to obtain the useful information from the signal or image that can be used for feature extraction stage. The Principal Component Analysis (PCA) is widely used in image processing, particularly in the area of face recognition and compression [22-25].

This study has addressed this issue and has presented a novel monitoring system for tool recognition and identification based on a comparative study between Discrete Wave Transformation (DWT) and Principal Component Analysis (PCA) combined with neural networks. The discrete wavelet transform (DWT) provides a time-frequency representation of the signal. The originality of this work comes from investigating visual and well as infrared images for detection the cutting tools and its health (normal or broken). It is one of the first studies that compare between visual and infrared for detection and evaluating the health of a 
milling process. In this paper Discrete Wave Transformation (DWT) and Principal Component Analysis (PCA) combined with neural networks will be used in a comparative study investigating the data obtained from infrared and visual imaging systems to detect the existence of the cutting tool and recognise its health conditions.

\section{Methodology}

Figure 1 presents the methodology used in this paper. Experiments are carried out on CNC milling machine. Images of the cutting tool are captured using an infrared camera and its associated visual camera during the process.

Captured images are then saved on a computer for further analysis using Matlab software. Image processing algorithms and two different methods namely PCA and DWT combined with neural networks are used to recognise the existing and the health condition of the cutting tool. The methodology, as shown in Figure 1, is to compare the two suggested approaches in order to develop an improved computer algorithm for tool recognition and evaluate between infrared and visual data.

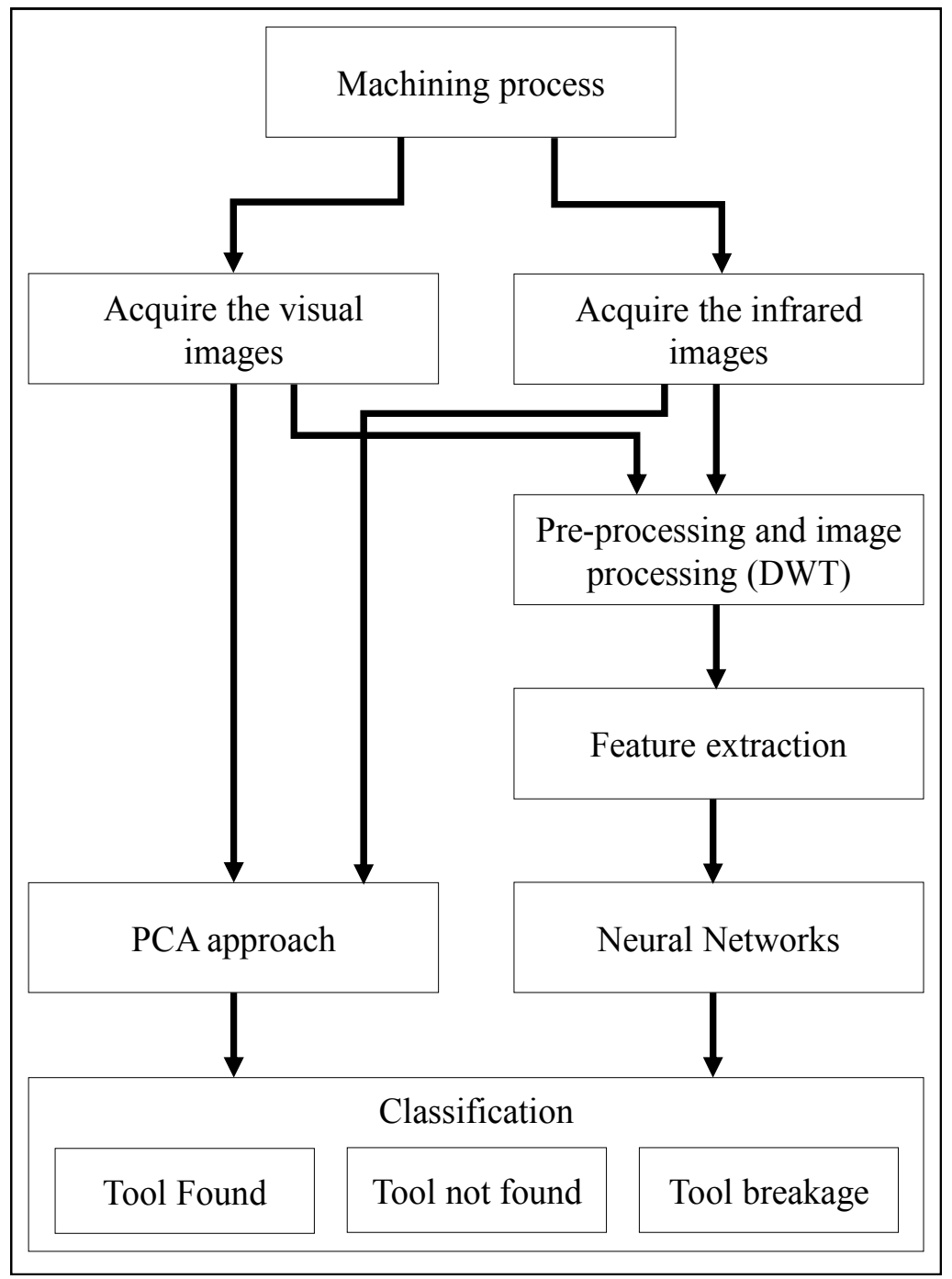

Figure 1: A schematic diagram of the research methodology implemented in this paper 


\subsection{The application of the PCA Approach}

This research work investigates the capability of the image processing and PCA approach to recognise the existing and the health condition of the cutting tool. Images are acquired using infrared and visual cameras.

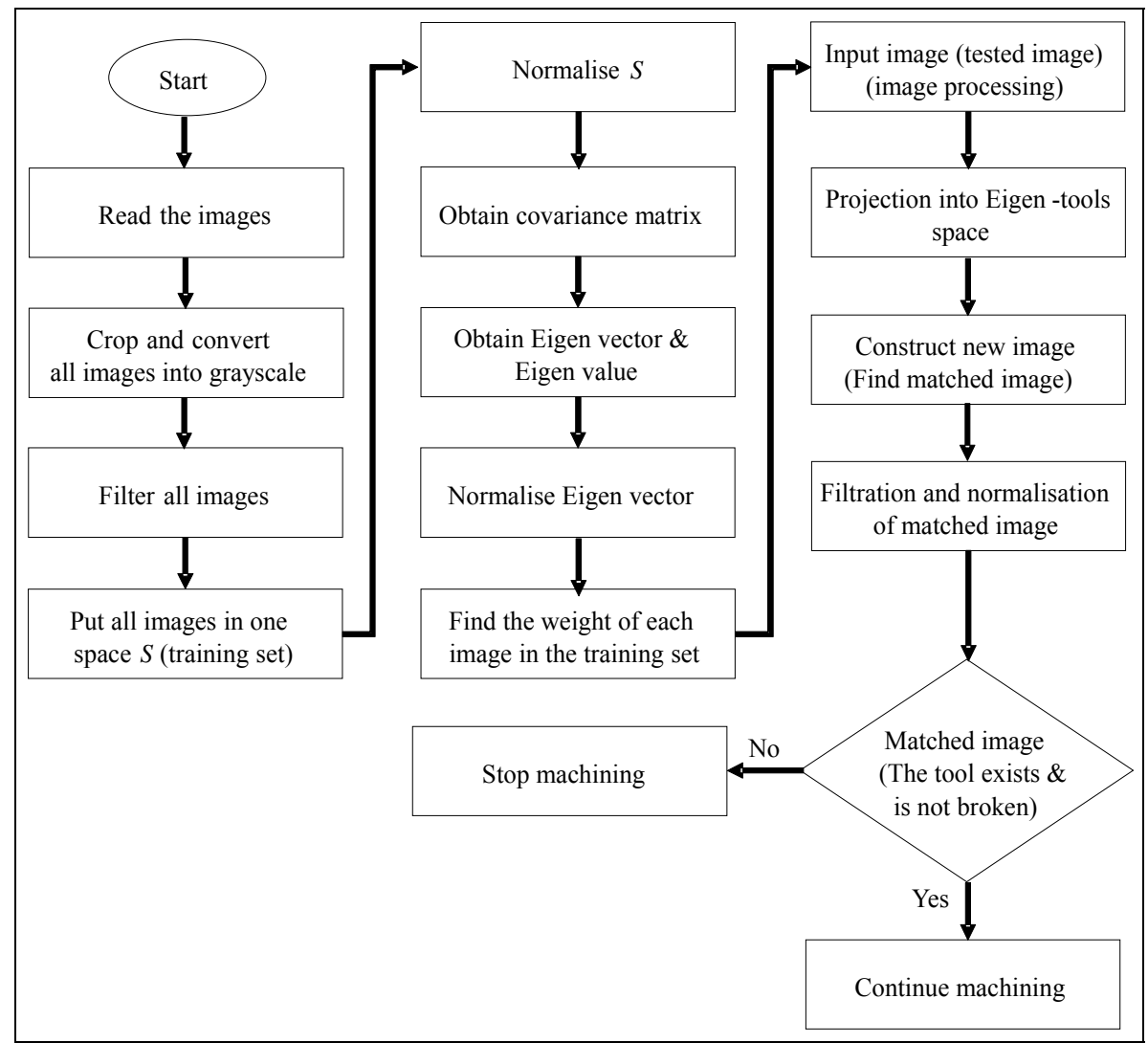

Figure 2: A detailed schematic diagram of the PCA approach

PCA approach is used in order to develop a computer algorithm for tool recognition. The PCA approach is used in this study because it is fast and relatively simple algorithm and has shown high performance in literature for other applications. Figure 2 presents the flowchart of the implemented algorithm where further technical details will be described further in the following sections. Infrared and visual images are captured before developing a grayscale image and cropping the images to the required area. Images are then filtered using median filter in order to remove noise from images. Median filter is used in this study as it is normally very effective in removing noise while preserving edges. It is particularly effective at removing 'salt and pepper' type noise. All images are then put in one space $S$ and normalised. The covariance matrix is then calculated where Eigen vector and Eigen value are obtained and normalised. The weight of each image in the training set is calculated. More details are described in section 3 of this paper.

\subsection{The application of DWT combined with Artificial Neural Networks (ANN)}

The acquired images by the machine vision system are saved to the computer and MATLAB software is used for image processing and data analysis. A feed-forward neural network is used in order to develop a pattern recognition method for tool recognition. The research methodology of using DWT with ANN is shown in Figure 3. The acquired images are converted into grayscale level in order to simplify and also, speed up the processing of these colour images. Noise removal is used for the purpose of image restoration in order to remove 
the noise from the image in such a way that the "original image" can be clearly discernible. Then, image gradient is introduced which is the directional change of the intensity or colour of the image. It is used to extract and convey additional information from the images as shown in Figure 4.

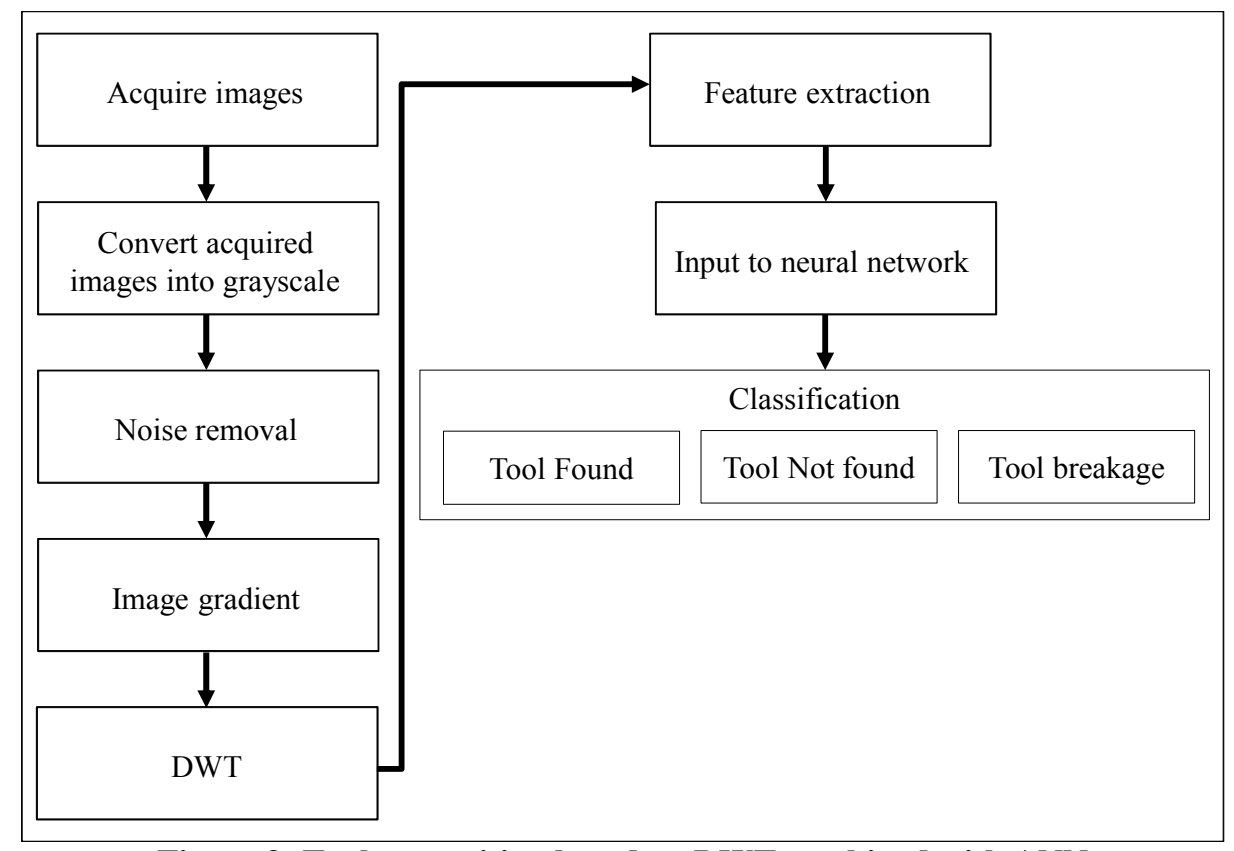

Figure 3: Tool recognition based on DWT combined with ANN

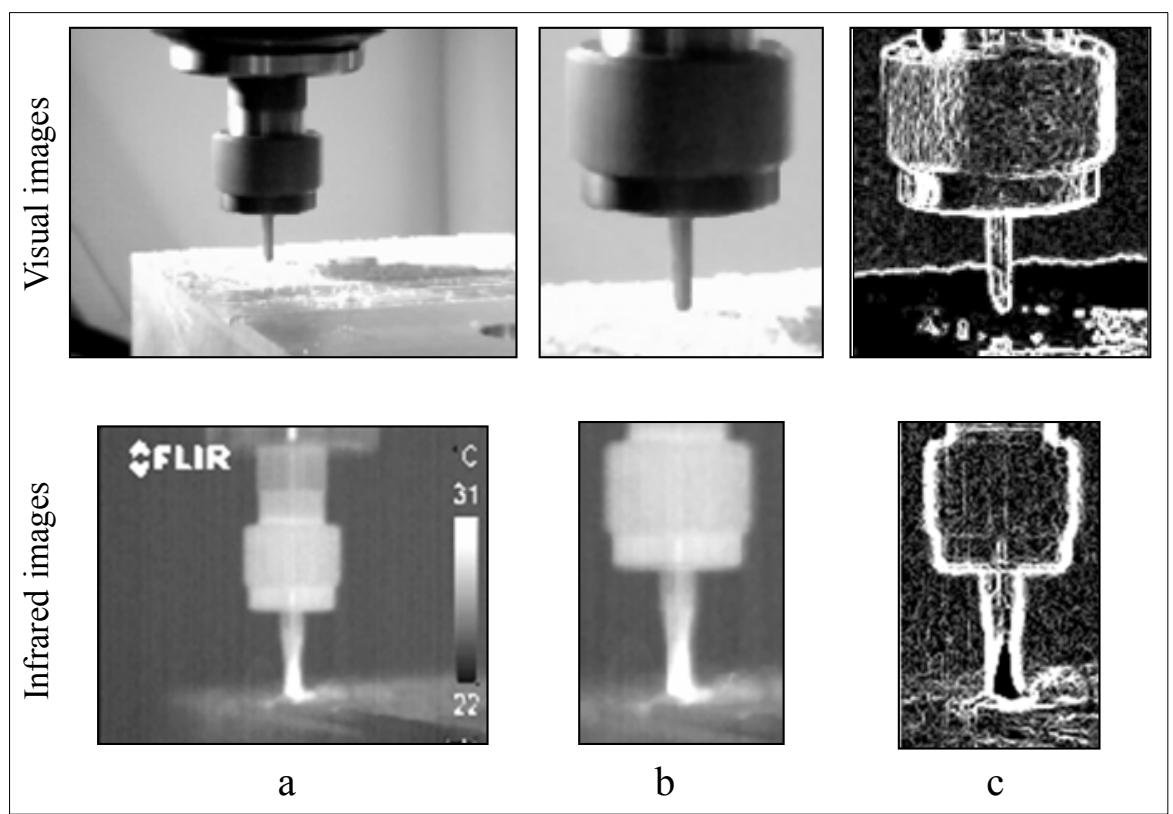

Figure 4: Visual and infrared images (a), region of interest (b) and gradient image (c)

The discrete wavelet transform (DWT) provides a time-frequency representation of the signal. It is implemented in order to reduce the required computational time and resources. DWT has an advantage of being easy to use. It also, provides flexible multi-resolution analysis of an image [19-21]. DWT used in signal and image processing in order to obtain the useful information from the signal or image that can be used for feature extraction stage. A feature extraction stage is introduced as a significant procedure to be used in order to extract 
important information from all images which have a significant effect on the condition to be predicted. The obtained two images after applying DWT are used for feature extraction stage. Four features are applied; standard division, average, kurtosis and skewness. These features are obtained from both images of low-pass and high-pass filters. As a result eight features are used to input to the neural network for tool recognition and classification. A pattern recognition stage; feed-forward back-propagation network is used for pattern recognition and tool recognition and classification. The use of ANNs is confined to distinguish between three conditions: 'tool found, 'tool not found' and 'tool breakage'. Further details regarding the used image processing, feature selection and pattern recognition are described in section 8 .

\section{Principle of PCA approach for tool recognition}

Normally an image is constructed as a 2D or 3D matrix, however; from mathematical view it can be represented as vector with the length $w \times h$, where the rows of the image are placed beside each other [26] as shown in Figure 5. Where:

$a_{0,0}, a_{0,1}, \ldots, a_{0, N-1}, a_{1,0}, a_{1,1}, \ldots, a_{1, N-1}, \ldots, a_{M-1,0}, \ldots, a_{M-1, N-1}$ are elements of the image shown in Figure 5. The number of elements of this image is $w \times \mathrm{h}$. Each image of the training set is then represented in a vector image and then placed in one space with dimension of $w \times h$, which known as image space (training set). The same set should be normalised. Figure 6 presents training set and its normalised set. All tool vectors are placed in a very narrow cluster in the image space.

Next, covariance matrix of the set of tool images (image space) is obtained, after which the Eigenvector is calculated. Eigenvectors form a set of features that characterises the variation between images of the tool for different conditions. Thus, each of the tool images contributes more or less to the eigenvector, in which the eigenvectors can be displayed as a set of ghostly tools, which are called Eigen-tools as shown in Figure 7. Images in the training set can be approximately reconstructed by storing a small collection of weights for each tool and a small set of standard pictures. So tool images in a training set can be the weighted sums of small collection of characteristics of the tools. An effective model should be able learn and recognise particular tool to build the characteristic features from known images of the tool. Then it should be capable in recognising the particular tool by comparing features of the image with known features of the images in the training set.

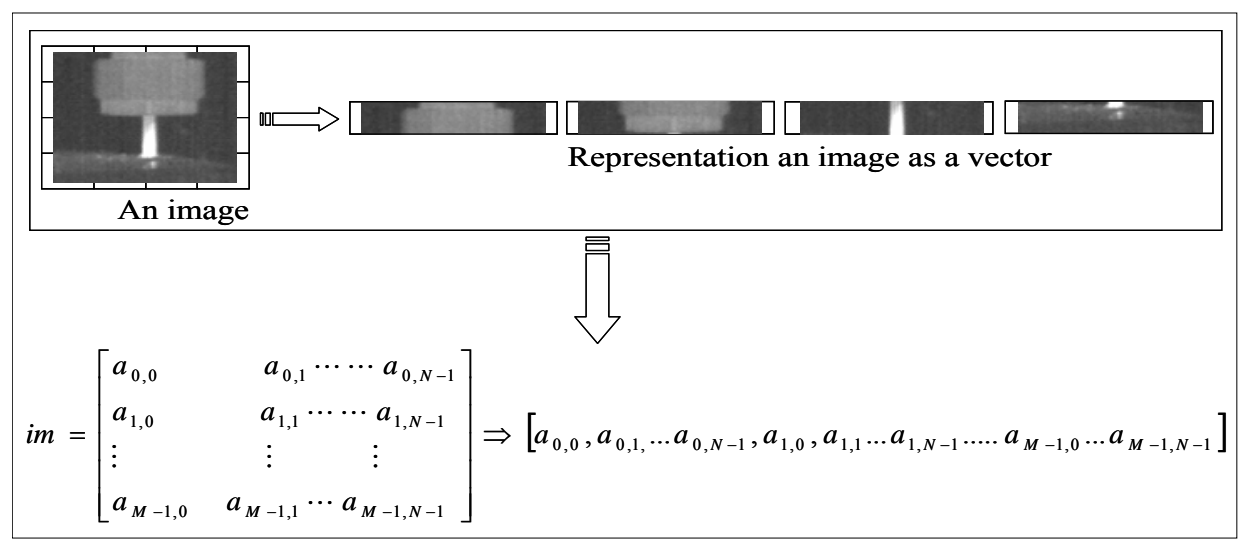

Figure 5: Representation of an image as vector image 


\subsection{Mathematical analysis of PCA approach}

First step is to obtain a set $S$ with $m$ images, where $m$ in this study is 28 tool images (see Figure 6). Each image is cropped into size (241x176); and then transformed into a vector of size $v(42416 \times 1)$ and then located into the set $S$, as shown in equation (1).

$$
S=\left\{\Gamma_{1}, \Gamma_{2}, \Gamma_{3}, \ldots, \Gamma_{n}\right\}
$$

where:

$S$ is the training set with size $S(42416 \times 28)$ and;

$\Gamma$ is the vector that represent an image of the training set.

Once the set $S$ has been obtained, all images will be normalised (see Figure 6) and then the next step is to obtain the covariance matrix. Obtaining the covariance matrix $A$ by equation (2).

$$
A=\frac{1}{M} \sum_{n=1}^{M}\left(x_{i}-\bar{x}\right)\left(x_{i}-\bar{x}\right)
$$

where:

$$
\begin{aligned}
& A=A^{\prime} \\
& X_{i} \text { is the row vector } \\
& A=\left[v_{1}, v_{2}, v_{3}, \ldots v_{n}\right]
\end{aligned}
$$

where (vi) is eigenvector.

By calculating $L$ as in equation (4)

$$
L=A^{*} A^{\prime}
$$

The eigenvector $(v)$ and the eigenvalue $(\lambda)$ can be obtained using equation (5):

$$
L v=\lambda v
$$

where:

$\lambda$ is the eigenvalue $>1$ and $v$ is the eigenvector. $\lambda_{k}$ can be calculated as:

$$
\lambda_{k}=\frac{1}{M} \sum_{k=1}^{M}\left(u_{k}^{T} \Gamma_{k}\right)^{2}
$$

The eigenvector $(v)$ can be normalised which will be the Eigen-images of the images of the training set, also called Eigen-tools or ghostly tools (see Figure 7).

An input image, image $Q$, is then acquired for testing by converting the image into grayscale and cropped in the size of $Q(241 \times 176)$. Figure 7 illustrates the acquired and matched images and their representation in binary format. The input image is then reshaped into a vector $q$ of size (61401 x 1 pixels). 


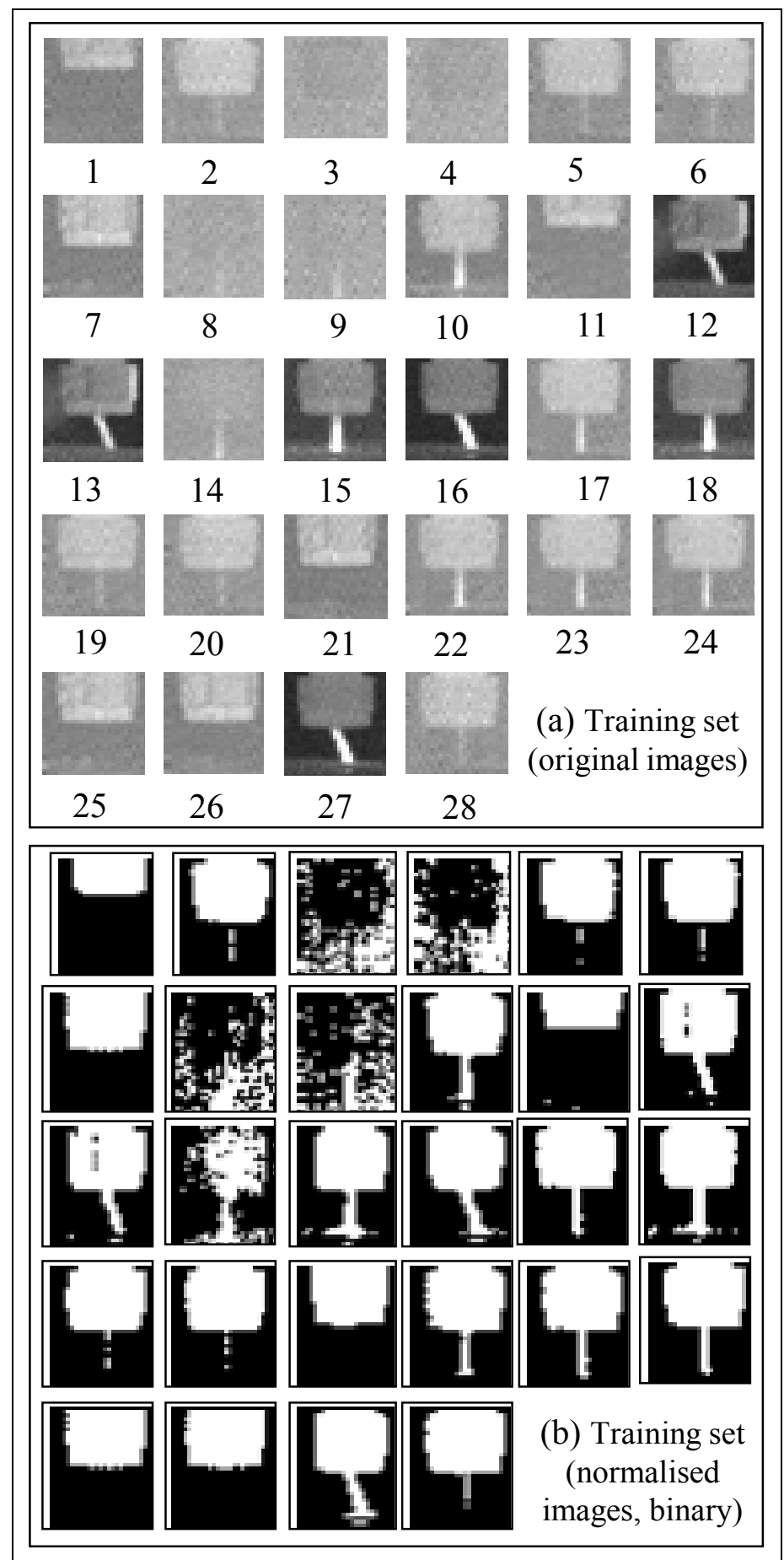

Figure 6: Images of cutting tools used as training set and its normalised images (binary) 


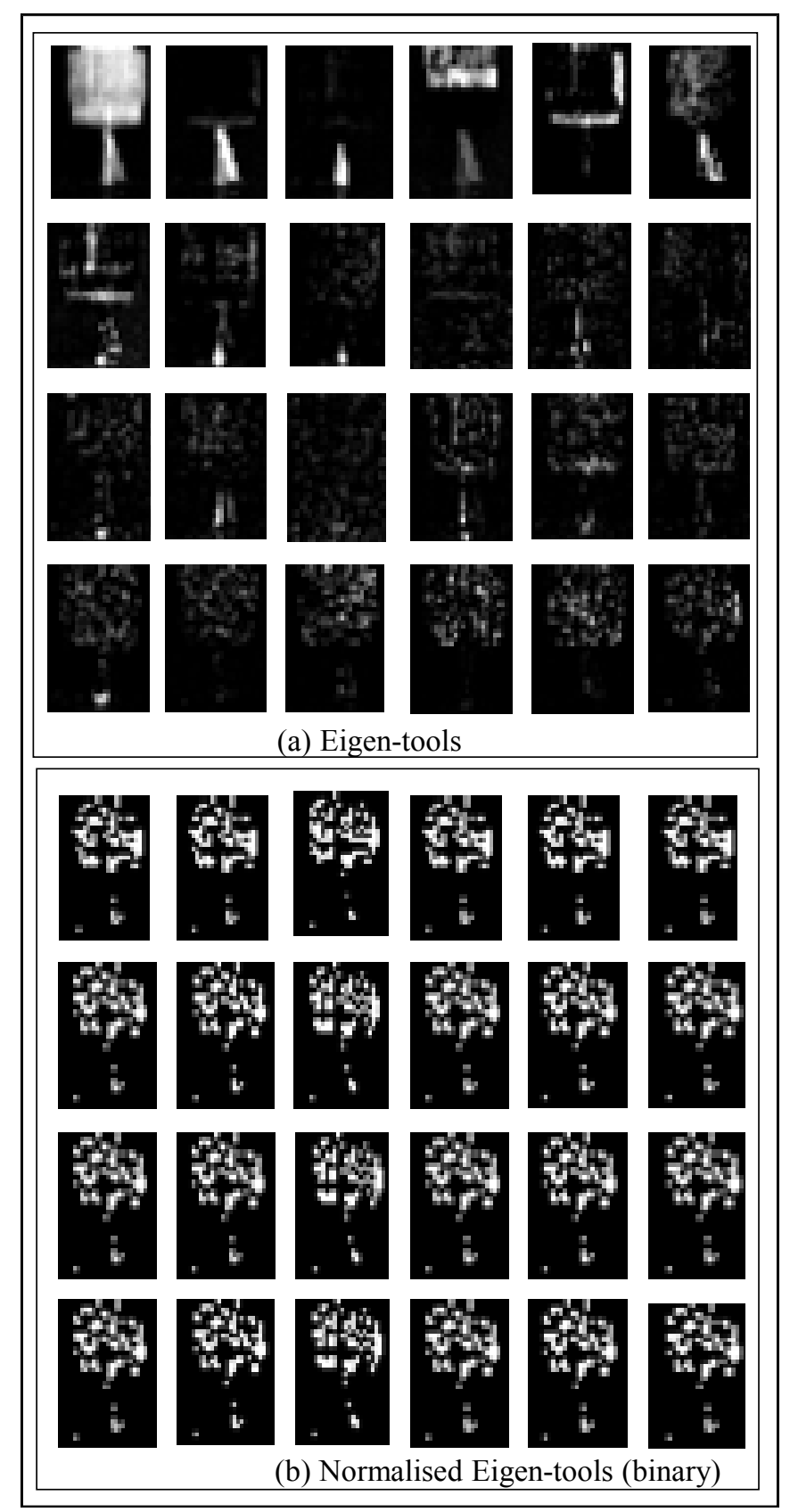

Figure 7: Eigen-tools and its normalised Eigen-tools (binary)

\subsection{Tool recognition procedure}

- Project the input image into each Eigen-tools of by calculating the dot product of eigenvector of input image $(q)$ and each eigenvector of Eigen-tools $(u)$ space.

$$
P=q^{*} u
$$

- Reconstruct a new image $R$ by summation of the mean $\Psi$ and multiplication of $u$, and $P$. reconstructed image should be the same size as the input image $R(241 \times 176)$. 


$$
R=\Psi+u^{*} p
$$

- Once the above procedure is completed, the system will show the reconstructed image (matched image), see Figure 6. If the reconstructed image matches an image of 'tool not found' or 'broken tool', a warring message will appear and the execution of the program will stop till the user takes a suitable action. This simply can be done by calculating the difference between the input image $Q(241 \times 176)$ and reconstructed image $R(241 \times 176)$.

The difference, $D=Q-R$

$$
\begin{aligned}
& M=\frac{1}{n} \sum_{i=1}^{n} D_{i} \\
& s t=\sqrt{\frac{\sum_{i=1}^{n} D_{i}^{2}}{n}}-\frac{\sum_{i=1}^{n} D_{i}}{n}
\end{aligned}
$$

The average $M$ and standard division $S T$ of the difference $(D)$ is then calculated. Each image is expected to have different values of $M$ and $S T$; if the value of an input image has matched the same value of a 'tool not found' or 'broken tool', the warring dialog box will appear with a short suitable massage and stop the machine tool.

\section{The application of DWT combined with neural networks}

Image reduction (IR) and analysis are required in order to extract the superlative information from the acquired images. Two types of image processing algorithms, namely gradient and discrete wavelet (DWT) are used. These techniques are integrated in a condition monitoring algorithm to evaluate their capabilities when used to process infrared and visual image features to recognise the existing and the health condition of the cutting tool.

\subsection{Image gradient technique}

Image gradient is the directional change of the intensity or color of the image. It widely used in image processing such as face to extract useful information from images. The magnitude $\Delta f(x, y) \mid$ and orientation $\theta_{G}$ of intensity gradient are computed as following [27-28]:

$$
\begin{aligned}
& |\Delta f(x, y)|=\sqrt{G_{x}(x, y)^{2}+G_{y}(x, y)^{2}} \\
& \theta_{G}(x, y)=\arctan \left(\frac{G_{y}(x, y)}{G_{x}(x, y)}\right)
\end{aligned}
$$

where

$$
\begin{aligned}
& G_{x}(x, y)=f(x, y) * K_{x(x, y)} \\
& G_{y}(x, y)=f(x, y) * K_{y(x, y)}
\end{aligned}
$$

where

* Denotes a 2D convolution operation and $K_{x(x, y)}$ and $K_{y(x, y)}$ are the horisontal and vertical gradient kernels, respectively.

In this study gradient technique is used to analysis the image in order to improve the visualisation of the image (see Figure 4). 


\subsection{Discrete wavelet transforms (DWT):}

The discrete wavelet transform (DWT) of an image is representing the image into other form. It does not change the information content present in the image. The Wavelet Transform provides a time-frequency representation of the signal. It used in order to reduce the computation time and resources required. DWT has an advantage easy to use and minimise the computation time and can be used for a finite group of data. It also, provides flexible multi-resolution analysis of an image [29, 30]. DWT is used in signal and image processing in order to obtain the useful information from the signal or image that can be used for further analysis. The input image is the region of interest of the original image of the cutting tool img (371 x 251) pixel.

\subsubsection{Principle of DWT}

Spouse signal $S(n)$ where $n=0,1,2,3, \ldots, M-1$ and $j \geq j_{0}$ the discrete wavelet transform can be defined as:

$$
\begin{gathered}
W \varphi\left(j_{0}, k\right)=\frac{1}{M} \sum_{n} S(n) \varphi_{j_{0, k}}(n) \\
W_{\psi}\left(j_{0} k\right)=\frac{1}{M} \sum_{n} S(n) \psi_{j, k}(n)
\end{gathered}
$$

where:

$n$ is a number of samples of signal

$j$ and $j_{0}$ are integers where $j \geq j_{0}$.

$\varphi_{j_{0, k}}$ and $\psi_{j, k}$ are the transformation kernel.

Having an image $\operatorname{img}(m, n)$ the decomposition will be applied in directions $m$ and $n$ (horizontally, vertically and diagonally respectively). The decomposition of the image $i m g(m$, $n)$ will be computes the approximation coefficients matrix $\varphi(m, n)$ and details coefficients matrices $\psi(m, n)$ obtained by wavelet decomposition of the input image. Firstly the matrix (image) decomposition of the processing of the image will be done by rows using wavelet (high-pass) and then scaling (low-pass) function followed by columns. The obtained coefficients as follows:

$$
\begin{aligned}
& \varphi(m, n)=\varphi(m) \varphi(n) \text { is the low-pass filter. } \\
& \psi(m, n)=\psi(m) \psi(n) \text { is the high-pass filter. }
\end{aligned}
$$

Feature selection is a significant procedure to be used in order to extract important information from all images which have a significant effect on the condition to be predicted. The obtained two images after applying DWT are used for feature extraction stage. Four features are applied; standard division, average, kurtosis and skewness. These features are obtained from both images of high-pass and low-pass filters. As a result eight features will be used to input to the neural network for tool wear classification. 


\section{Neural Network Structure}

MATLAB neural network toolbox is utilised to build the ANN models. The used ANN in this research work has feed forward structure within one hidden layer is used between the input and output layers and one output variable in the training data set. Computer memory in this study is sufficient for the training. Due to the choice of the feed-forward back-propagation network, the features are normalised in range between zero and one for the training phase. All features from the images of 'normal conditions', 'tool not found' and 'tool breakage' are gathered in a large cell array. Each row will represent the features of an eight features of an image of the tool.

The training can cease according to the criteria of either mean square error (MSE) reaches a certain value or the number of training epochs reaches a certain value. In this application, a target mean square error of $10^{-9}$ and a maximum iteration number (epoch) of 11550 is selected from the author's experience. The training process would stop if any of these conditions are met. The initial weights and biases of the network are generated randomly by the program. 


\section{The Experimental setup}

The system consists of an infrared camera, visual camera, computer and a machine tool as shown in the Figure. Infrared and visual cameras are used in order to locate and track the tool conditions. A typical infrared image of the tool with resolution of $441 \times 526 \times 3$ pixels and visual image of resolution of $480 \times 640 \times 3$ pixels are illustrated in the top left corner of Figure 8. An electrical magnetic stand is used to hold the cameras. It offers stability and flexibility to fix the camera by suitable angle during machining. A computer system with computer software (lab-windows/cvi, visual basic and lab-view) is used to acquire the images from the camera. Captured images are then saved in bmp format for further analysis. Image processing (MATLAB software) is used for further analysis.

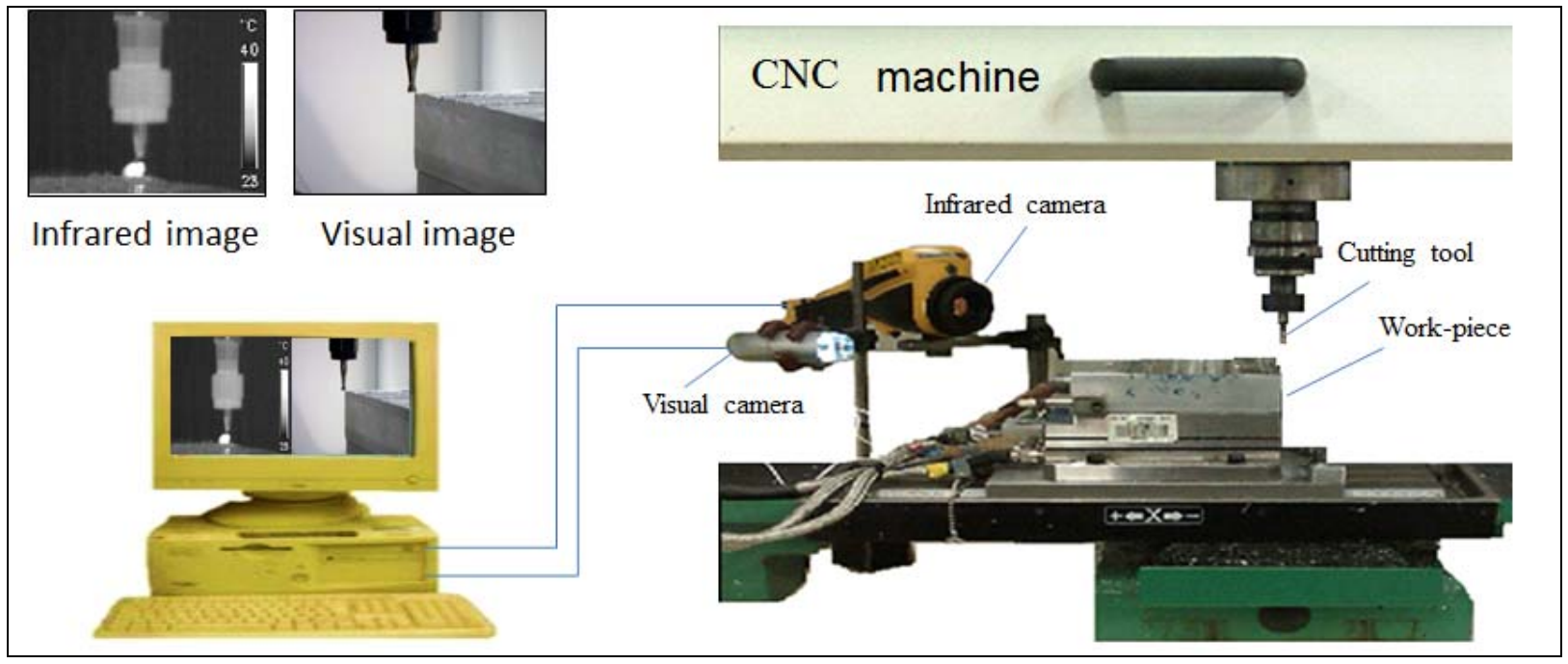

Figure 8: Experimental setup

\section{Evaluation of the performance of the PCA approach}

The PCA approach for tool recognition has been applied for both infrared and visual images. An 1864 of infrared images and 714 visual images are tested in this work. The tested results have shown a good performance of the developed program PCA approach.

\subsection{PCA approach using infrared images}

For the total of 1864 tested images, the PCA approach has recognised the tested images of infrared data with successes of $98.96 \%$. Incorrect evaluations are found to be 18 images from 1864. In some cases the wrong classification appears because of the resolution of the acquired image is relatively low as shown in Figure 9, where the tool is not well recognised. This is has occurred 18 times during execution of the program. 


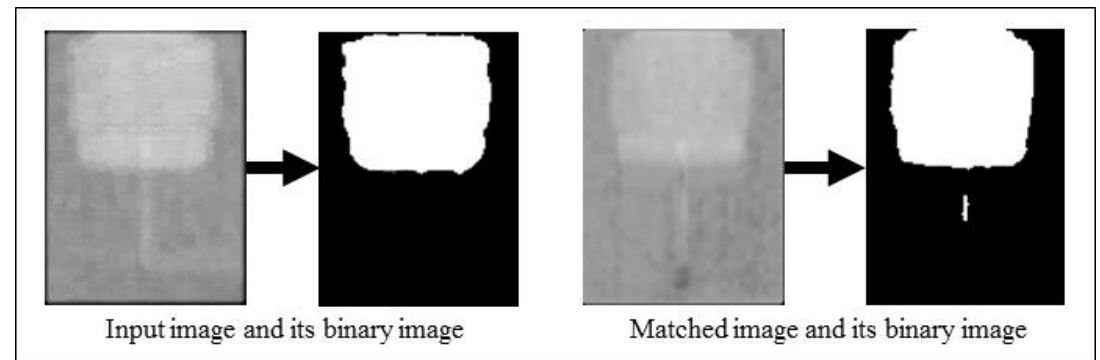

Figure 9: An example of the infrared input, matched images and their representation in binary of wrong estimation.

Figure\# presents examples of the acquired/tested images and the reconstructed images of the three conditions. Condition 1 shows the 'tool found', condition 2 is 'tool not found', while condition 3 shows the 'tool breakage'. Once the acquired image of 'tool found', 'tool not found' or 'tool breakage' matches the same image of the same condition in the training set, a suitable message will appear to notify the operator and the machine will stop. From Figure\# it can be clearly seen that the reconstructed image has correctly matched the acquired image of the same condition. From the above results and discussion, it can be clearly said that the developed program had successfully recognised and estimated the tool existence.

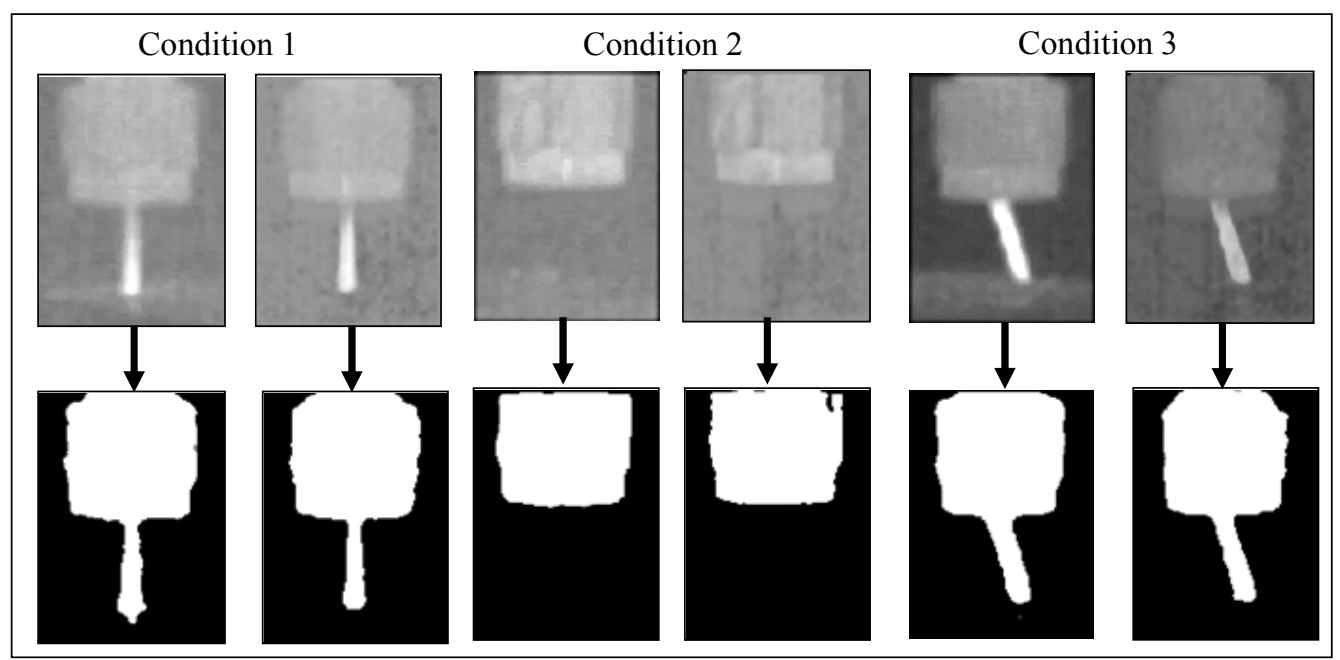

Figure\# 10: Examples of the three faulty conditions of the tool using infrared data (Tool found, Tool not Found, and Tool breakage)

\subsection{PCA approach using visual images}

A total of 714 images have been tested in this case. The tested results have shown that the PCA approach when applied for visual images has recognised the tested images, but with some noise in some cases as shown in Figure 8. The successes rate has been found to be $95.94 \%$ (29 incorrect classifications). From Figure 8, it can be seen that the matched and binary images $(b, c)$ contain some noise which makes it difficult to correctly analyse and categorise the image condition. 


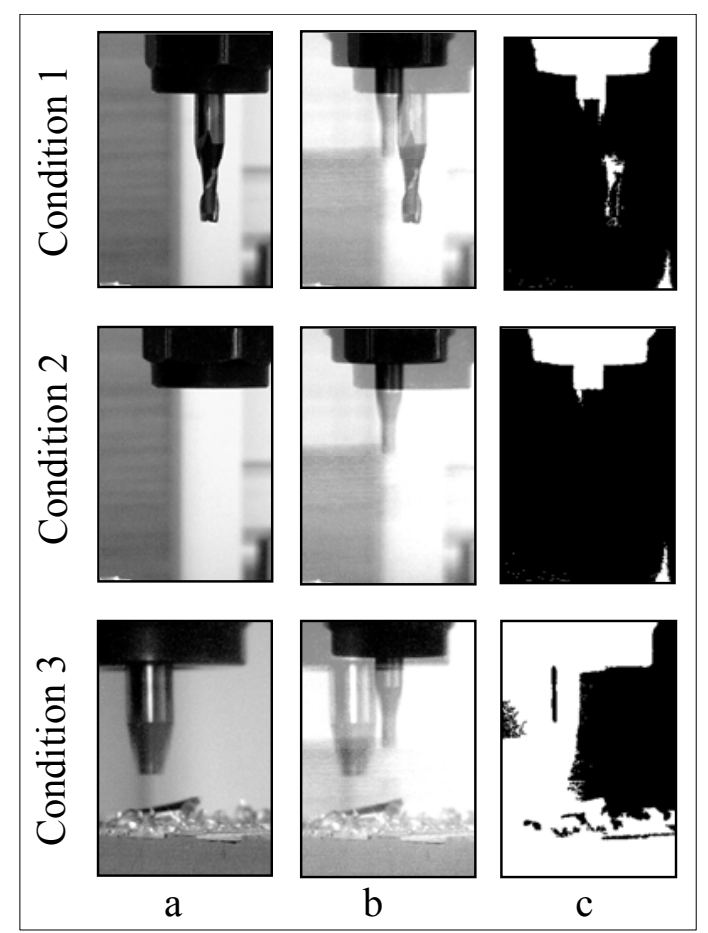

Figure 8: The input visual images (a), matched (b) and binary images (c) of visual images of three conditions: 'Tool found' (1), 'Tool not found' (2) and 'Tool breakage' (3)

\section{Evaluation of using DWT combined with neural networks}

Result and discussion in this section is divided two stages. First stage is to evaluate the ANN based on infrared image data and DWT. Second stage is to evaluate the ANN based on visual image data and DWT.

\subsubsection{Evaluation of DWT and ANN using Infrared Images}

In order to evaluate the performance of the ANN, training of the neural network has been done with 885 images, 230 images of 'normal conditions', 11 images of 'tool not found' and 44 images of 'tool breakage'. Eight features for each image are used as input to train the proposed neural network. The performance of tool wear estimation has been tested with 561 images of the tool of different experiments. The ANNs performance is compared with previously tools of known conditions. Figure 9, presents the actual evaluation of the tools of the training of the ANN (previously known conditions of the tool); and neural network estimation trial 1 of 'normal conditions', trial 2 of 'tool not found', and trial 3 'tool breakage'. The pattern recognition approach has correctly estimated all tested images with $100 \%$ success. 


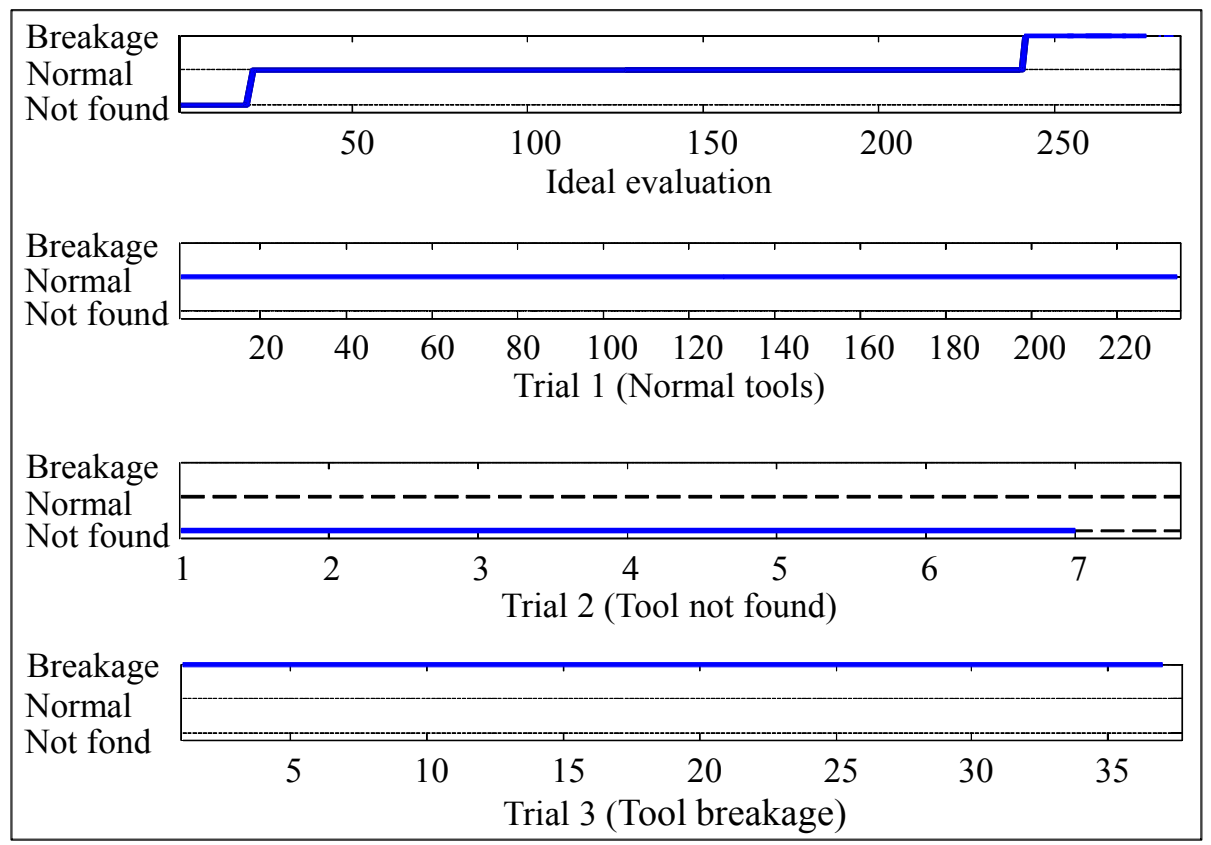

Figure 9: Tool recognition using pattern recognition approach based on infrared image data

\subsection{2. $\quad$ Evaluation of DWT and ANN using Visual Images}

Similar to the previous section, a feed-forward neural network is used. Training of the neural network has been performed with 227 images; 216 images of 'normal conditions', 5 images of 'tool not found' and 6 images of 'tool breakage'. Eight features for each image are used as inputs to train the neural network. The performance of tool wear estimation has been tested with 561 images of the tool of different experiments. The ANNs performance is compared with previously tools of known conditions. Figure 10, presents the actual evaluation of the tools of the training of the ANN); and neural network estimation condition 1 of 'normal conditions', condition 2 of 'tool not found' and condition 3 of 'tool breakage'. The pattern recognition approach has estimated all tested images with $89.63 \%$ successes as shown in Figure 11. 


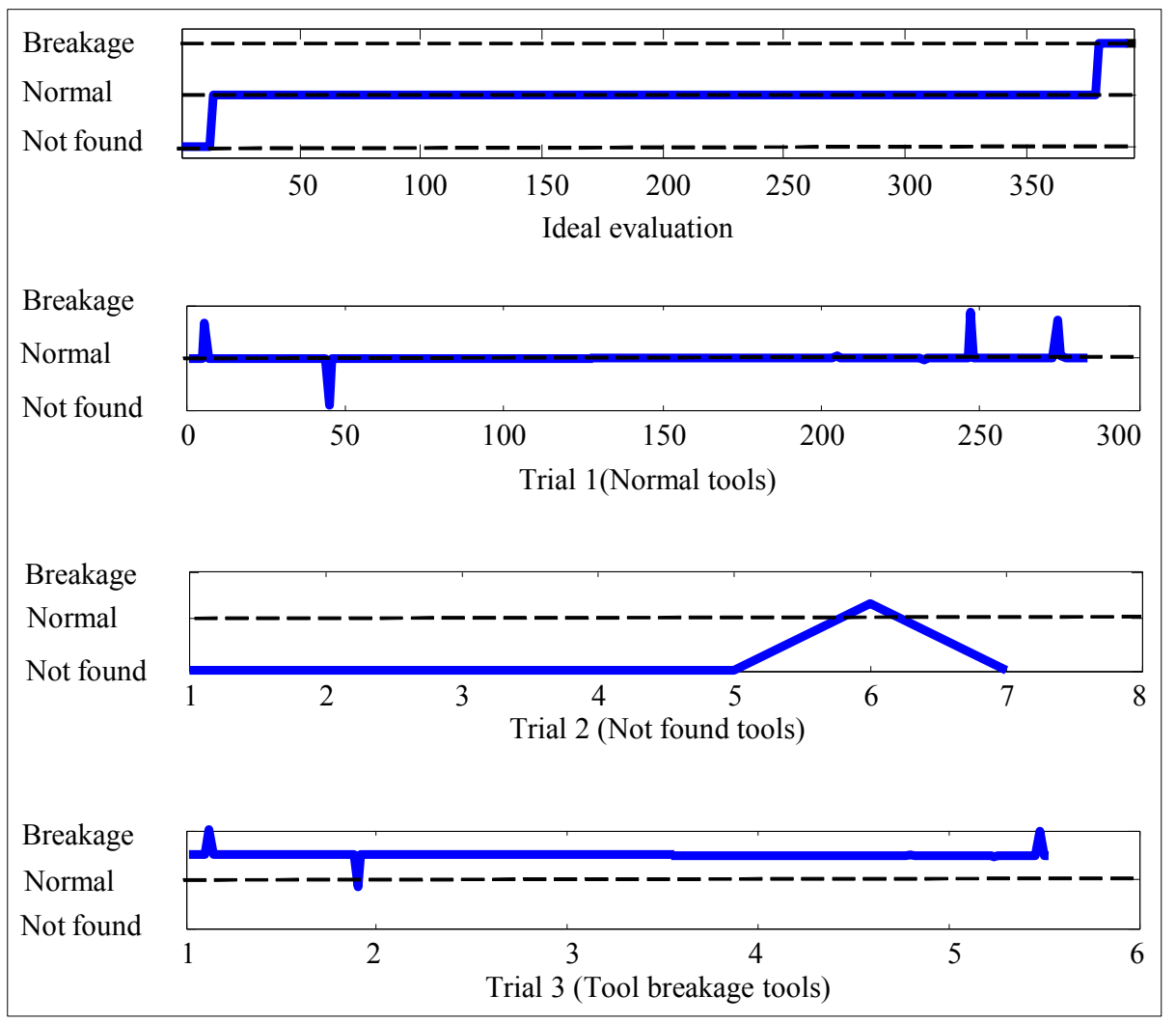

Figure 10: Tool recognition using pattern recognition approach based on visual image data

\section{Conclusion}

One of the primary functions of computerised condition monitoring systems in machining operations is to identify the existence of the cutting tool before starting any machining process. This paper has presented a novel comparison study for the recognition of tool conditions. It examined the use of infrared and visual image data, with image processing using PCA and DWT combined with neural networks to verify the condition of the cutting tool in an end milling process (i.e. normal or broken). Figure 11 presents a comparison between the two approaches for the two types of data. The results show that the use of infrared data processed using DWT and the obtained features processed using neural networks has produced $100 \%$ success rate. However, the use of visual data for the same techniques shows much less success rate. Same characteristic is also shown by the use of PCA where the infrared data has shown improved results in comparison to the visual data. The results of this paper show that the infrared data, combined with suitable image processing and artificial intelligence could be a very efficient method for on-line condition monitoring of cutting tool conditions in machining operations. The advantage of this technique is that that the infrared and vision systems could be able to detect in real time any problems with the tool including non-existence of the tool or during the moment of breakage which is difficult to achieve by common methods. In addition, positioning of the infrared and visual cameras are much easier to maintain the monitoring process of the tool. 


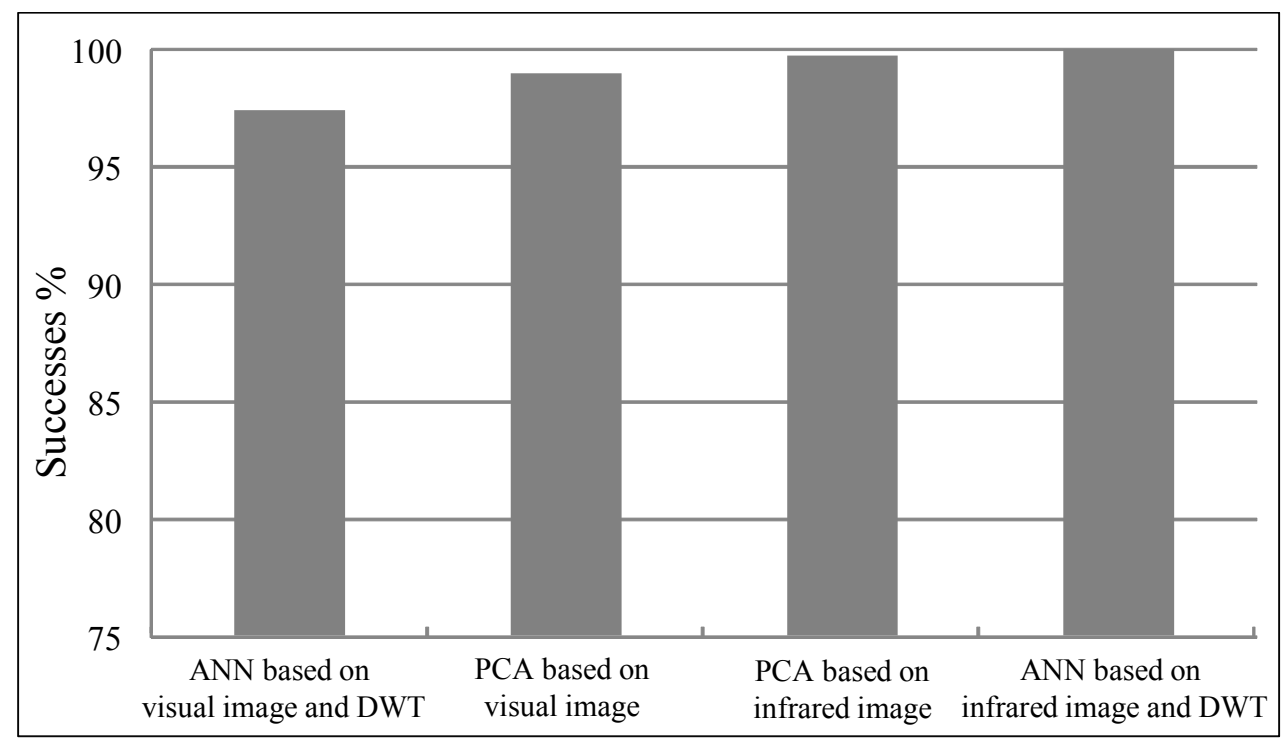

Figure 11: A comparison between the two suggested approaches using visual and infrared data.

\section{References}

[1] A. Al-Habaibeh, G. Liu, and N. Gindy, "Sensor fusion for an integrated process and machine condition monitoring system," 15th Triennial World Congress of the International Federation of Automatic Control, July 21-26 2002, Barcelona, Spain.

[2] A. Al-Habaibeh and N. Gindy, "Self-Learning Algorithm for Automated Design of Condition Monitoring Systems for Milling Operations", The International Journal of Advanced Manufacturing Technology, Vol 18, Issue 6, pp 448-459, 2001.

[3] A. Al-Habaibeh, F. Zorriassatine, and N. Gindy, "Comprehensive Experimental Evaluation of a Systematic Approach for Cost Effective and Rapid Design of Condition Monitoring Systems Using Taguchi's Method", Journal of Materials Processing Technology , 124(3) , June 2002, pp 372-383, ISSN 0924-0136.

[4] M. Rizal, J.A. Ghani, M. Z. Nuawi and C. H. C. Haron, "A Review of Sensor System and Application in Milling Process for Tool Condition Monitoring," vol. 7, no. 10, pp. 2083-2097, 2014.

[5] T. I. Ogedengbe, R. Heinemann, and S. Hinduja, "Feasibility of Tool Condition Monitoring on Micro-Milling Using Current Signals," vol. 14, no. 3, pp. 161-172, 2011.

[6] U. Zuperl, F. Cus, and J. Balic, "Intelligent Cutting Tool Condition Monitoring In Milling," vol. 49, no. 2, pp. 477-486, 2011.

[7] C. Bisu, A. Gerard, M. Zapciu, and O. Cahuc, "The Milling Process Monitoring Using 3D Envelope Method," Adv. Mater. Res., vol. 423, pp. 77-88, Dec. 2011.

[8] Girardin, F., D. Remond and J.F. Rigal, (2010). "A New Method for Detecting Tool Wear and Breakage in Milling.” Int. J. Mater. Forming, 3(1): 463-466. 
[9] Kious, M., A. Ouahabi, M. Boudraa, R. Serra and A. Cheknane, (2010). "Detection Process Approach of Tool Wear in High Speed Milling.” Meas., 43: 1439-1446.

[10] Figliola, R.S. and D.E. Beasley, (2000). "Theory and Design for Mechanical Measurements". 3rd Edn. John Wiley and Sons, Inc., New York.

[11] Chuangwen, X., L. Zhe and L. Wencui, (2009). "A Frequency Band Energy Analysis of Vibration Signals for Tool Condition Monitoring." Proceeding of International Conference on Measuring Technology and Mechatronics Automation.

[12] Kalvoda, T. and Y.R. Hwang, (2010). "A Cutter Tool Monitoring in Machining Process using Hilbert.” Int. J. Mach. Tool. Manu., 50: 495-501.

[13] J. C. Su, C. K. Huang, and Y. S. Tarng, "An Automated Flank Wear Measurement of Microdrills Using Machine Vision,” J. Mater. Process. Technol., vol. 180, no. July, pp. 328-335, 2006.

[14] M. Malekian, S. S. Park, and M. B. G. Jun, "Tool Wear Monitoring of Micro-Milling Operations," J. Mater. Process. Technol., vol. 209, no. 10, pp. 4903-4914, Jun. 2009.

[15] A. V. Atli, O. Urhan, S. Ertürk, and M. Sönmez, "A computer Vision-Based Fast Approach to Drilling Tool Condition Monitoring," Proc. Inst. Mech. Eng. Part B J. Eng. Manuf., vol. 220, no. 9, pp. 1409-1415, Jan. 2006.

[16] H. H. Shahabi, T. H. Low, and M. M. Ratnam, "Notch Wear Detection in Cutting Tools Using Gradient Approach and Polynomial Fitting,” Int. J., pp. 1057-1066, 2009.

[17] S. Bagavathiappan, B. B. Lahiri, T. Saravanan, J. Philip, and T. Jayakumar, "Infrared Physics \& Technology Infrared Thermography for Condition Monitoring - A review," Infrared Phys. Technol., vol. 60, pp. 35-55, 2013.

[18] Amin Al-Habaibeh and Robert Parkin, "An autonomous low-Cost infrared system for the online monitoring of manufacturing processes using novelty detection", The International Journal of Advanced Manufacturing Technology, Volume 22, Numbers 3-4, Sept 2003, SpringerVerlag London Ltd, ISSN: 0268-3768.

[19] A. Al-Habaibeh, F. Shi, N. Brown, D. Kerr, M. Jackson, and R. M. Parkin, “A Novel Approach for Quality Control System Using Sensor Fusion of Infrared and Visual Image Processing For Laser Sealing of Food Containers," Measurement Science and Technology, vol. 15, no. 10. pp. 1995-2000, 01-Oct-2004. 
[20] C.H. Lauro, L.C. Brandão, D. Baldo, R.A. Reis, J.P. Davim, Monitoring and processing signal applied in machining processes - A reviewReview Article

Measurement, Volume 58, December 2014, Pages 73-86

[21] R. J. Colom-Palero, R. Gadea-Girones, F. J. Ballester-Merelo, and M. Martínez-Peiro, "Flexible architecture for the implementation of the two-dimensional discrete wavelet transform (2D-DWT) oriented to FPGA devices," Microprocess. Microsyst., vol. 28, no. 9, pp. 509-518, Nov. 2004.

[22] A. N. Akansu, W. a. Serdijn, and I. W. Selesnick, "Emerging Applications of Wavelets: A review," Phys. Commun., vol. 3, no. 1, pp. 1-18, Mar. 2010.

[23] S. Asadi, C. D. V. S. Rao, and V. Saikrishna, "A Comparative Study of Face Recognition with Principal Component Analysis and Cross-Correlation Technique," Int. J. Comput. Appl., vol. 10, no. 8, pp. 17-21, Nov. 2010.

[24] J. Gan, D. Zhou, and C. Li, "A Method For Improved PCA in Face Recognition," Int. J. Inf. Technol. Vol. 11 No. 11 2005, vol. 11, no. 11, pp. 79-85, 2005.

[25] S. S. Panda, G. Deepthi, and V. Anisha, "Face Recognition Using PCA and Feed Forward Neural Networks," Int. J. Comput. Sci. Telecomm., vol. 2, no. 8, pp. 79-82, 2011.

[26] S. Kaymak, "Face Detection , Recognition and Reconstruction Using Eigenfaces," 2003.

[27] H. Park, T. Schoepflin, and Y. Kim, "Active Contour Model with Gradient Directional Information : Directional Snake,” vol. 11, no. 2, pp. 252-256, 2001.

[28] Ju Han and Bir Bhanu, "Individual Recognition Using Gait En- Ergy Image,", IEEE Transactions on Pattern Analysis and Ma- chine Intelligence, pp. 316-322, 2006.

[29] P. Chaovalit, A. Gangopadhyay, G. Karabatis, and Z. Chen, "Discrete Wavelet Transform-Based Time Series Analysis and Mining," vol. 43, no. 2, 2011.

[30] V.Galiano, O Lopez-Granado, M P Malumbres, L A Drummond and H. Migallon. "GPU-based 3D lower tree wavelet video encoder. "EURASIP Journal on Advances in Signal Processing 2013.1 (2013): 1-13. 